\title{
First Principle Local Density Approximation Description of the Electronic Properties of Ferroelectric Sodium Nitrite
}

\author{
C. E. Ekuma, M. Jarrell, and J. Moreno \\ Departmentof Physics \& Astronomy, and Center for Computation \& Technology, Louisiana \\ State University (LSU) \\ Baton Rouge, Louisiana 70803, USA \\ L. Franklin, G. L. Zhao, J. T. Wang, and D. Bagayoko \\ Department of Physics, Southern University and A\&M College in Baton Rouge (SUBR) Baton \\ Rouge, Louisiana 70813, USA
}

The electronic structure of the ferroelectric crystal, $\mathrm{NaNO}_{2}$, is studied by means of firstprinciples, local density calculations. Our ab-initio, non-relativistic calculations employed a local density functional approximation (LDA) potential and the linear combination of atomic orbitals (LCAO). Following the Bagayoko, Zhao, Williams, method, as enhanced by Ekuma and Franklin (BZW-EF), we solved self-consistently both the Kohn-Sham equation and the equation giving the ground state charge density in terms of the wave functions of the occupied states. We found an indirect band gap of $2.83 \mathrm{eV}$, from $\mathrm{W}$ to R. Our calculated direct gaps are 2.90, 2.98, $3.02,3.22$, and $3.51 \mathrm{eV}$ at $\mathrm{R}, \mathrm{W}, \mathrm{X}, \Gamma$, and $\mathrm{T}$, respectively. The band structure and density of states show high localization, typical of a molecular solid. The partial density of states shows that the valence bands are formed only by complex anionic states. These results are in excellent agreement with experiment. So are the calculated densities of states. Our calculated electron effective masses of $1.18,0.63$, and $0.73 \mathrm{~m}_{\mathrm{o}}$ in the $\Gamma-\mathrm{X}, \Gamma-\mathrm{R}$, and $\Gamma-\mathrm{W}$ directions, respectively, show the highly anisotropic nature of this material.

Pacs Numbers: 77.84.-s, 71.20.-b, 71.20.Mq, 71.20.Nr

\section{INTRODUCTION AND MOTIVATION}

Sodium nitrite is one of the $\mathrm{ABO}_{2}$ group with several interesting properties such as ferroelectricity, paraelectricity and piezoelectricity. The ground state of $\mathrm{NaNO}_{2}$ has one of the simplest structures in the $\mathrm{ABO}_{2}$ group. Since the discovery of its ferroelectric property in 1958, by Sawada et al. [1], there has been increased interest in diverse properties of $\mathrm{NaNO}_{2}$, ranging from structural changes to dielectric, electronic, thermal, electrical and elastic properties, as well as its non-linear optical spectra [2]. Despite this noted interest in ferroelectric $\mathrm{NaNO}_{2}$, there has been very little study of the electronic band structure of the crystal [3].

One of the earliest electronic band gap measurements of ferroelectric $\mathrm{NaNO}_{2}$ was by Asao et al. [4] who found band gaps of $2.3,2.6,3.1$, and $3.7 \mathrm{eV}$ for different current directions in their resistivity work. Also, using the same method, Takagi and Gesi [5] found a band gap of $2.4 \mathrm{eV}$. These authors did not specify whether the gaps in question are direct or indirect. The infrared absorption spectra study of Sidman [6] at a temperature of $77 \mathrm{~K}$ gave a band gap of 
$3.22 \mathrm{eV}$ while that of Verkhovskaya and Sonin [7], at $293.15 \mathrm{~K}$, led to a value of $3.14 \mathrm{eV}$. The semi-empirical LCAO work of El-Dib and Hassan [8], for room temperature (293.15 K), found a band gap of $3.24 \mathrm{eV}$. The scanning electron microscopy, diffuse reflectance spectroscopy, and electrical measurements study of Balabinskaya et al. [9], at a temperature of $300 \mathrm{~K}$, reported an absorption edge of $3.0 \mathrm{eV}$.

One of the earliest theoretical studies of the band structure of ferroelectric $\mathrm{NaNO}_{2}$ was the $\mathrm{X} \alpha(\alpha=0.75)$ exchange calculation of Kam et al. [10] who found a direct band gap of 3.45 $\mathrm{eV}$ at $\Gamma$ and an indirect band gap of $2.0 \mathrm{eV}$. The Full-potential linear muffin-tin orbital (FPLMTO) method of Ravindran et al. [2] found an indirect band gap of $2.2 \mathrm{eV}$. The generalized gradient approximation (GGA) work of Wang et al. [11] utilized a full-potential linearized augmented plane wave (FL-APW) method to calculate a band gap of less than $2.1 \mathrm{eV}$. The LDA approach of Zhuravlev and Korabel'nikov [12], using the nonlocal Troullier-Martins (TM) pseudopotentials and the Slater exchange potential with a correlation correction, led to a direct band gap of $2.50 \mathrm{eV}$ at $W$. Zhuravlev and Poplavnoi [13], also using nonlocal Troullier-Martins (TM) pseudopotentials and the Slater exchange $(\alpha=1)$ potential with a correlation correction, found a minimum forbidden band gap of $3.07 \mathrm{eV}$. The LDA result of Jiang et al. [14], using an orthogonalized linear combination of atomic orbitals (OLCAO) method, found an indirect band gap of $2.95 \mathrm{eV}$ from $S$ to $R$ symmetry points. The periodic Hatree-Fock (PHF) method of McCarthy [15] overestimated the gap by a factor of 2-3. Henkel et al. [16] employed their plane-wave and Gaussian basis set and an X $\alpha$ exchange and reported a direct band gap of 2.70 $\mathrm{eV}$. These results suffice to see that theoretical calculations have not resolved the under- or overestimation of the band gap of ferroelectric $\mathrm{NaNO}_{2}$.

In light of the preceding overview, the aim of this study is to attempt to obtain the measured, fundamental band gap and other electronic properties of ferro- $\mathrm{NaNO}_{2}$ with ab-initio, self-consistent LDA calculations. The confirmation of our predictions of the band gaps and other properties for $\mathrm{BaTiO}_{3}$ [17], $\mathrm{TiO}_{2}$ [18], CdS [19], c- $\mathrm{Si}_{3} \mathrm{~N}_{4}$ [20], c-InN [21], and $\mathrm{SrTiO}_{3}$ [22] is a basis for the above presumption. Further, the mathematical rigor of the method [18-24] suffices to expect it to lead to much better results.

\section{METHOD OF CALCULATION}

Our calculations employed the Ceperley and Alder [25] local density functional potential as parameterized by Vosko, Wilk, and Nusair [26]. We implemented the linear combination of atomic orbitals (LCAO), using Gaussian functions for the radial parts. We utilized a program package developed at the Ames Laboratory of the US Department of Energy (DOE), in Ames, Iowa [27]. Our calculations are non-relativistic and were performed using low temperature (120 $\mathrm{K})$ lattice constants. The distinctive feature of our approach resides in the implementation of the Bagayoko, Zhao, and Williams (BZW) method, as enhanced by Ekuma and Franklin (BZW-EF), consisting of concomitantly solving self-consistently two coupled equations as explained below. One of these equations is the Schrödinger type equation of Kohn and Sham [28], referred to as the Kohn-Sham (KS) equation. The second equation, which can be thought of as a constraint on the KS equation, is the one giving the ground state charge density in terms of the wave functions of the occupied states. 
The essentials of the method follow. Beginning with a minimum or small basis set capable of accounting for all the electrons in the system under study, one performs ab-initio, self-consistent calculations. This minimum or small basis set is constructed using orbitals resulting from selfconsistent calculations for the atomic or ionic species in the solid. As noted below, these species are $\mathrm{Na}^{1+}, \mathrm{N}^{1+}$, and $\mathrm{O}^{2-}$ for $\mathrm{NaNO}_{2}$. Subsequently, this basis set is augmented with one orbital and self-consistent calculations are done. This new orbital and others that may be added later are outputs of the self-consistent studies of the ionic species. The occupied energies of the two calculations for the solid are compared numerically and graphically. These occupied energies from Calculations I and II are generally different. A third calculation is carried out after adding another "ionic" orbital to the basis set. We note that adding an orbital means augmenting the size of the basis set by $2,6,10$, or 14 , depending on the $s, p, d$, or $f$ character of it, respectively. Again, the occupied energies from Calculations II and III are compared. This process continues until the occupied energies from a calculation, i.e., $\mathrm{N}$, are found to be identical to those from Calculation $(\mathrm{N}+1)$ immediately following it, within our computational uncertainties of $50 \mathrm{meV}$ or less. At that point, the calculations are completed and the results from Calculation $\mathrm{N}$ represent the physical description of the system under study. The basis set for Calculation $\mathrm{N}$ is referred to as the optimal basis set. Calculation $(\mathrm{N}+1)$ and others, with larger basis sets that contain the optimal one, provided linear dependency is avoided, reproduce the occupied energies from Calculation N. However, by virtue of the Rayleigh theorem [19,23], these calculations produce some unoccupied eigenvalues that are lower than those from Calculation $\mathrm{N}$, due to a non-trivial basis set and variational effect [23].

All the self-consistent calculations known to us carry out the iterative procedure that involves both the Kohn-Sham equation and the equation giving the ground state charge density. However, the methodical increase of the size of the basis set, as done in the BZW-EF method, entails changes in the radial and angular characteristics of the basis set as well as an increase in its size. It is in this sense that the BZW-EF method solves self-consistently the two equations in question to obtain, in a verified fashion, the minima of the occupied energies. The difference between our method and single trial basis set calculations may be best understood by noting that any serious radial, angular, or basis size deficiencies that may exist in a single trial basis set cannot be remedied by the iterative process noted above. In the case of the BZW-EF method, such deficiencies are corrected as the methodical augmentation of the basis set is carried out. The attainment of the minima of the occupied states signifies that there is no deficiency left to correct. When a larger basis set that contains the optimal one is utilized to carry out a calculation, the charge density, the potential, and the Hamiltonian resulting from self-consistency, as well as the occupied eigenvalues, are the same as the corresponding ones obtained with the optimal basis set. Hence, the Rayleigh theorem applies to the outputs of this calculations whose occupied energies are the same as those obtained with the optimal basis set. However, some unoccupied energies from the larger basis set are generally lower than (or equal) to their corresponding ones obtained with the optimal basis set. The lowering of these unoccupied energies cannot be ascribed to physical interactions, as the Hamiltonian did not change. It results from the non-trivial and well-defined basis set and variational effect.

The enhancement of the original BZW method is in the methodical increment of the basis set. This enhancement leads to adding the polarization $(p, d$, or $f)$ orbitals, for a given principal quantum number, before adding the spherically symmetric $s$ orbital (see Table 1). These additional unoccupied orbitals are needed to accommodate the reorganization of the electron cloud, 
including possible polarization, in the crystal environment. For valence electrons in molecules to solids, polarization has primacy over spherical symmetry.

While there are no specific rules governing the order of adding orbitals, our experience has been that orbitals often need to be added to the heaviest element before the others. Among these others, preliminary studies of charge transfer serve as a significant guide. In the case of $\mathrm{NaNO}_{2}$, even though oxygen is a little heavier than nitrogen, the neon configuration oxygen approaches after gaining 2 electrons intimate more stability than that of $\mathrm{N}^{1+}$. The ultimate aim of the addition of orbitals (i.e., basis functions) is to allow an optimal reorganization of the electronic cloud in the solid environment as compared to atomic or ionic ones.

Computational details germane to a replication of our work follow. Ferroelectric sodium nitrite (ferro- $\mathrm{NaNO}_{2}$ ) has an orthorhombic structure [29]. It is in the space group $\mathrm{C}_{2 \mathrm{v}}^{20}$ - IMM2, with space group number 44 and Patterson symmetry Immm [30]. The ferro- $\mathrm{NaNO}_{2}$ unit cell contains eight atoms: four (4) cations and four (4) anions whose positions are as indicated between parentheses: $\mathrm{Na}:(0,0.5881,0),(0.5,0.0881,0.5) ; \mathrm{N}:(0,0.1224,0),(0.5,0.6224,0.5)$; and O: $(0,0,0.1962),(0.5,0.5,0.6962),(0,0,0.8038),(0.5,0.5,0.3038)$.

We carried out five (5) different calculations utilizing five (5) different basis sets in search of the optimal one. Table 2 contains the basis sets utilized in the five (5) self-consistent calculations. Methodical increases of the basis set led to calculation IV as the one with the optimal basis set; i.e., the calculation yielding the minima of all the occupied energies. Calculation $\mathrm{V}$ does not lower any occupied energies as compared to Calculation IV. Hence, the electronic structure and related properties presented here were obtained with basis set IV, the optimal basis set.

Our self-consistent computations were performed utilizing experimental lattice constants of $3.518 \AA, 5.535 \AA$ and $5.382 \AA$ for $a, b$, and $c$, respectively, as obtained at $120 K$ [31]. Neutral charge calculations $\left(\mathrm{Na}^{0}, \mathrm{~N}^{0}\right.$ and $\left.\mathrm{O}^{0}\right)$ were carried out. Calculated charges were found to be +1 for $\mathrm{Na},+1$ for $\mathrm{N}$ and -2 for $\mathrm{O}$. Then, we performed self-consistent calculations for $\mathrm{Na}^{1+}, \mathrm{N}^{1+}$ and $\mathrm{O}^{2-}$ to get the input quantities for the calculations for $\mathrm{NaNO}_{2}$. The radial parts of the atomic wave functions were expanded in terms of Gaussian functions, employing a set of even-tempered Gaussian exponents.

The self-consistent atomic calculations provided trial atomic potentials for $\mathrm{Na}, \mathrm{N}$, and $\mathrm{O}$, respectively. These potentials were used to construct the initial potential for ferro- $\mathrm{NaNO}_{2}$. We used 16 Gaussian functions for the $s$ and $p$ states and 14 for the $d$ states for $\mathrm{Na}$ and $\mathrm{N}$, respectively. We utilized 17 Gaussian functions for the $s$ and $p$ states and 15 for the $d$ states for O. A mesh of $48 k$ points, with proper weights in the irreducible Brillouin zone, was employed in the self-consistent calculations. A total of 121 weighted k-points were used in band structure calculations and a total of 147 weighted k-points were employed to generate the energy eigenvalues for the electronic density of state calculation. The computational error for the valence charge was about 0.073343 for 52 electrons, a little more than $10^{-3}$ per electron. The self-consistent potentials converged to a difference around $10^{-5}$ after about 60 iterations.

\section{CALCULATED ELECTRONIC STRUCTURE}

Our calculated, ab-initio, self-consistent bands for ferro- $\mathrm{NaNO}_{2}$ (Calculation IV) are as shown in Fig. 1 and the comparison plot of the electron energy bands for basis set IV (solid lines) and basis set $\mathrm{V}$ (dashed lines) are as shown in Fig. 2. As is apparent in this graph, the eigenvalues 
of the occupied states totally converged, within computational errors of about $0.050 \mathrm{eV}$, showing clearly that Calculation IV is the one with the optimal basis set.

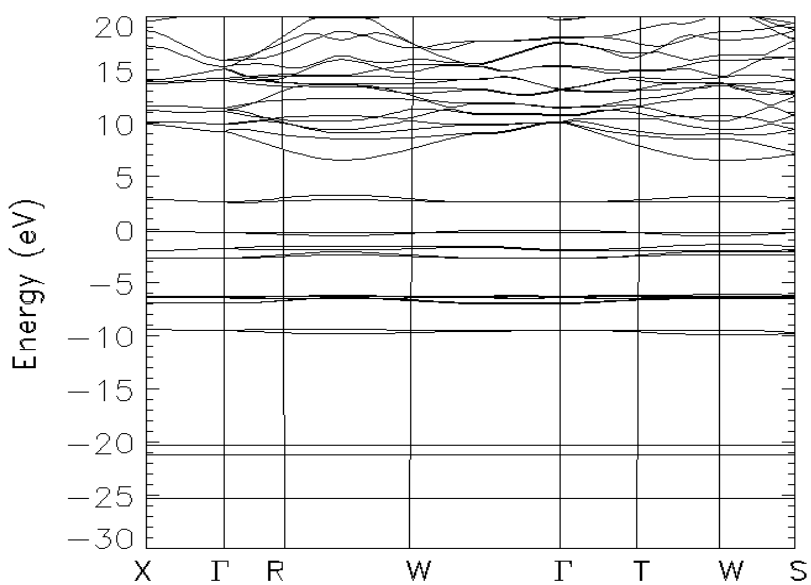

Fig. 1. The calculated electronic energy bands of ferro- $\mathrm{NaNO}_{2}$, from Calculation IV.

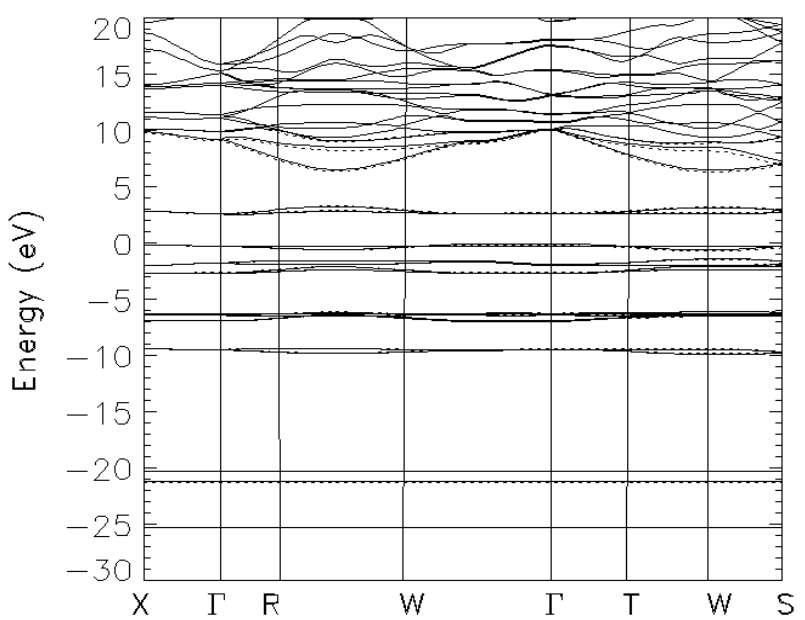

Fig. 2. The calculated electronic energy bands of ferro- $\mathrm{NaNO}_{2}$, from Calculations IV (full lines) and V (dashed lines). The occupied energies from Calculations IV and V are practically identical. 


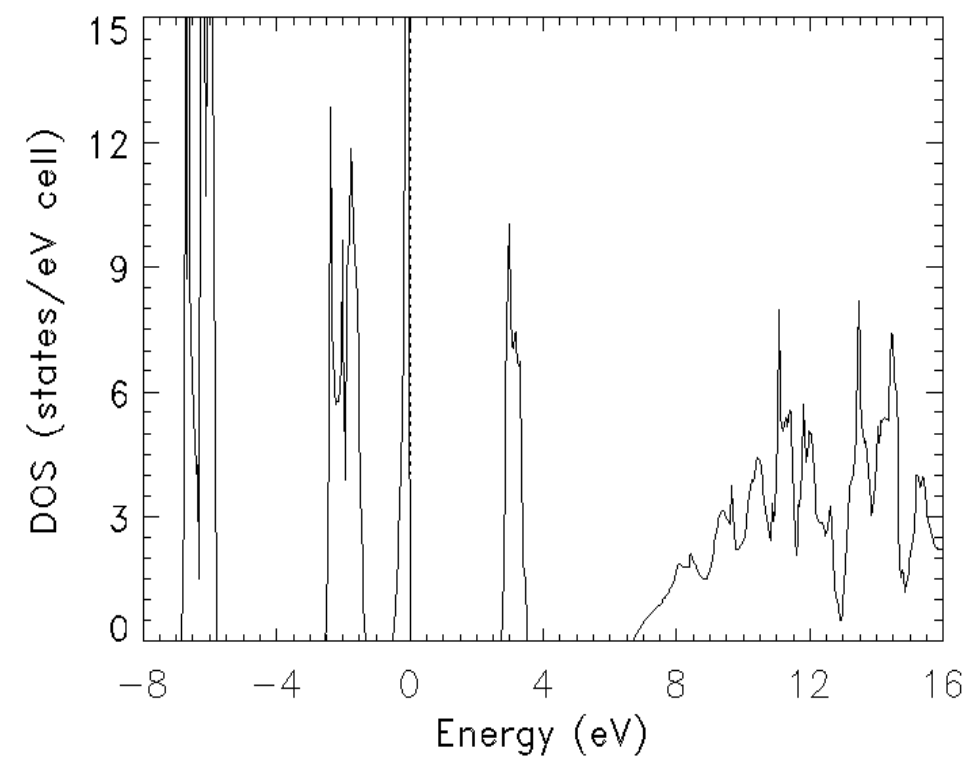

Fig. 3. The calculated density of states (DOS) of ferro- $\mathrm{NaNO}_{2}$, obtained with the BZW optimal basis set (i.e. Calculation IV).

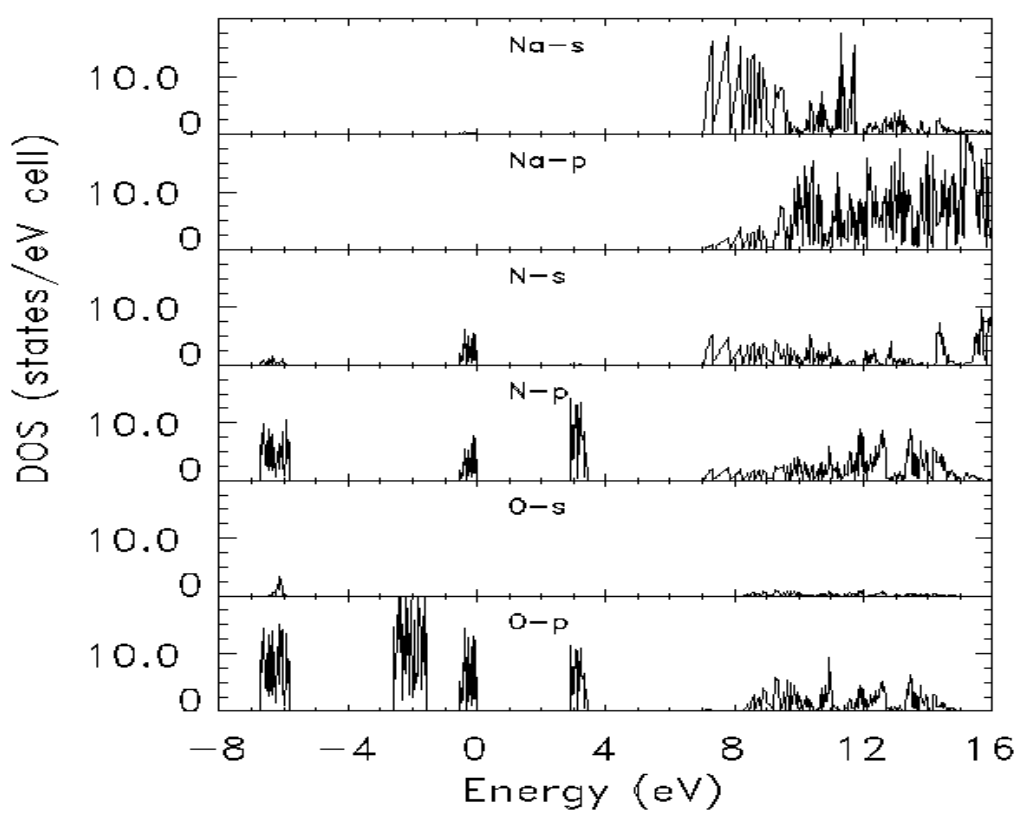

Fig. 4. The calculated partial density of states (pDOS) of ferro- $\mathrm{NaNO}_{2}$ as obtained with the BZW optimal basis set (i.e. Calculation IV). 
Table 1. Search for the optimal basis sets (Orbital added is in bold), as per the BZW method, for the description of the valence states of ferroelectric sodium nitrite (ferro- $\mathrm{NaNO}_{2}$ ). The optimal basis set is that from Calculation IV. Na is Sodium, N is Nitrogen, and O is Oxygen. The maximum of the Valence band occurred at $W$, while the minimum of the conduction band occurred at $R . \mathrm{NaNO}_{2}$ is an indirect band gap material.

\begin{tabular}{|c|c|c|c|c|c|c|c|}
\hline $\begin{array}{c}\text { Basis } \\
\text { Set }\end{array}$ & $\begin{array}{c}\mathrm{Na}, \mathrm{N} \\
\text { and } \mathrm{O}_{2} \\
\text { Core }\end{array}$ & Na Valence & $\begin{array}{c}\mathbf{N} \\
\text { Valence }\end{array}$ & $\mathrm{O}_{2}$ Valen & $\begin{array}{l}\text { Total No. } \\
\text { cof Valence } \\
\text { orbitals }\end{array}$ & \multicolumn{2}{|c|}{ Band Gap (eV) } \\
\hline & & & & & & $\begin{array}{c}\text { Direct at } \\
\Gamma\end{array}$ & Indirect \\
\hline I & $1 \mathrm{~s}$ & $2 \mathrm{~s} 2 \mathrm{p} 3 \mathrm{~s}$ & $2 \mathrm{~s} 2 \mathrm{p}$ & $2 \mathrm{~s} 2 \mathrm{p}$ & 34 & 2.40 & 2.38 \\
\hline II & $1 \mathrm{~s}$ & $2 \mathrm{~s} 2 \mathrm{p} 3 \mathrm{~s} 3 \mathbf{p}$ & $2 \mathrm{~s} 2 \mathrm{p}$ & $2 \mathrm{~s} 2 \mathrm{p}$ & 40 & 2.70 & 2.53 \\
\hline III & $1 \mathrm{~s}$ & $2 \mathrm{~s} 2 \mathrm{p} 3 \mathrm{~s} 3 \mathrm{p}$ & $2 \mathrm{~s} 2 \mathrm{p} 3 \mathrm{~s}$ & $2 \mathrm{~s} 2 \mathrm{p}$ & 42 & 3.18 & 2.93 \\
\hline$I V$ & $1 \mathrm{~s}$ & $2 s 2 p 3 s 3 p 3 d$ & $2 s 2 p 3 s$ & $2 s 2 p$ & 52 & 3.22 & 2.83 \\
\hline $\mathrm{V}$ & $1 \mathrm{~s}$ & $2 \mathrm{~s} 2 \mathrm{p} 3 \mathrm{~s} 3 \mathrm{p} 3 \mathrm{~d}$ & $2 \mathrm{~s} 2 \mathrm{p} 3 \mathrm{~s}$ & $2 \mathrm{~s} 2 \mathrm{p} 3 \mathrm{~s}$ & 56 & 3.24 & 2.83 \\
\hline
\end{tabular}

Table 2. The Calculated Eigenvalues (in eV) at the High Symmetry Points for ferro- $\mathrm{NaNO}_{2}$. The eigenvalues are obtained by setting the energy at the top of the valence band, which occurred at $W$, equal to zero.

\begin{tabular}{|r|r|r|r|r|r|r|r|}
\hline \multicolumn{7}{|c|}{ X } \\
\hline-25.11 & -25.11 & -21.03 & -21.03 & -21.01 & -21.01 & -20.97 & -20.97 \\
\hline-20.05 & -20.05 & -9.24 & -9.24 & -6.73 & -6.73 & -6.21 & -6.21 \\
\hline-6.08 & -6.08 & -2.51 & -2.51 & -1.80 & -1.80 & -0.01 & -0.01 \\
\hline 3.01 & 3.01 & 10.08 & 10.08 & 10.36 & 10.36 & 11.38 & 11.38 \\
\hline 11.79 & 11.79 & 13.96 & 13.96 & 14.12 & 14.12 & 14.32 & 14.32 \\
\hline 17.49 & 17.49 & 18.90 & 18.90 & 19.82 & 19.82 & 24.92 & 24.92 \\
\hline 30.26 & 30.26 & & & & & & \\
\hline \multicolumn{7}{|c|}{$\Gamma$} & \multicolumn{7}{|c|}{$\Gamma$} & & \\
\hline-25.11 & -25.09 & -21.13 & -21.03 & -21.02 & -21.01 & -20.89 & -20.89 \\
\hline-20.13 & -20.07 & -9.68 & -9.28 & -6.34 & -6.32 & -6.3 & -6.16 \\
\hline-6.00 & -5.89 & -2.04 & -1.76 & -1.70 & -1.33 & -0.51 & -0.10 \\
\hline 3.12 & 3.51 & 6.66 & 8.59 & 9.31 & 9.61 & 10.31 & 10.91 \\
\hline 12.53 & 13.59 & 13.86 & 13.97 & 13.97 & 14.67 & 14.78 & 16.26
\end{tabular}




\begin{tabular}{|c|c|c|c|c|c|c|c|}
\hline 16.47 & 18 & 18.84 & 20.17 & 20.43 & 20.86 & 20.96 & 21.97 \\
\hline 22.06 & 31.43 & & & & & & \\
\hline \multicolumn{8}{|c|}{$\mathrm{R}$} \\
\hline-25.15 & -25.15 & -21.00 & -21.00 & -20.99 & -20.99 & -20.92 & -20.92 \\
\hline-20.18 & -20.18 & -9.44 & -9.44 & -6.74 & -6.74 & -6.14 & -6.14 \\
\hline-6.08 & -6.08 & -2.38 & -2.38 & -1.47 & -1.47 & -0.07 & -0.07 \\
\hline 2.83 & 2.83 & 9.18 & 9.18 & 10.12 & 10.12 & 11.02 & 11.02 \\
\hline 12.02 & 12.02 & 13.4 & 13.4 & 14.46 & 14.46 & 15.62 & 15.62 \\
\hline 15.73 & 15.73 & 17.96 & 17.96 & 22.35 & 22.35 & 24.19 & 24.19 \\
\hline 24.99 & 24.99 & & & & & & \\
\hline \multicolumn{8}{|c|}{$\mathrm{W}$} \\
\hline-25.15 & -25.15 & -21.02 & -21.02 & -21.00 & -21.00 & -20.98 & -20.98 \\
\hline-20.06 & -20.06 & -9.28 & -9.28 & -6.84 & -6.84 & -6.13 & -6.13 \\
\hline-6.08 & -6.08 & -2.41 & -2.41 & -1.79 & -1.79 & 0 & 0 \\
\hline 2.98 & 2.98 & 10.31 & 10.31 & 10.39 & 10.39 & 10.93 & 10.93 \\
\hline 11.59 & 11.59 & 13.38 & 13.38 & 13.50 & 13.50 & 15.72 & 15.72 \\
\hline 17.68 & 17.68 & 18.34 & 18.34 & 19.96 & 19.96 & 24.09 & 24.09 \\
\hline 26.91 & 26.91 & & & & & & \\
\hline \multicolumn{8}{|c|}{$\mathrm{T}$} \\
\hline-25.14 & -25.14 & -21.08 & -21.08 & -21.01 & -21.01 & -20.89 & -20.89 \\
\hline-20.11 & -20.11 & -9.53 & -9.53 & -6.31 & -6.31 & -6.15 & -6.15 \\
\hline-6.06 & -6.06 & -1.91 & -1.91 & -1.52 & -1.52 & -0.23 & -0.23 \\
\hline 3.28 & 3.28 & 7.33 & 7.33 & 9.74 & 9.74 & 10.95 & 10.95 \\
\hline 12.71 & 12.71 & 13.11 & 13.11 & 14.43 & 14.43 & 16.59 & 16.59 \\
\hline 17.99 & 17.99 & 18.98 & 18.98 & 19.60 & 19.60 & 20.71 & 20.71 \\
\hline 25.00 & 25.00 & & & & & & \\
\hline
\end{tabular}

Table 2 shows the energies at the high symmetry points. Figs. 3 and 4 show the calculated, total (DOS) and partial (pDOS) density of states derived from the bands in Fig. 1. The calculated peaks in the valence band DOS closest to the Fermi energy are at $-0.25 \pm 0.1 \mathrm{eV}$ and $-2.4 \pm 0.2 \mathrm{eV}$. We found a third peak at $-5.8 \pm 0.1 \mathrm{eV}$ which agrees rather well with the experimental value of $-5.8 \mathrm{eV}$ reported by Kamada et al. [32]. In the conduction band, we found relatively broad peaks whose centers are located at 8.25, 9.50, 10.50, 11.25, and 12.00 $\mathrm{eV}$, respectively. The widths of these broad peaks are about $0.5 \mathrm{eV}$. Caution is required in using the conduction band density of states at energies above $6 \mathrm{eV}$. As explained by Jin et al. [33] and Bagayoko et al. [24, 34], BZW-EF calculations are concerned with correctly solving 
equations describing the ground states. Hence, while the low-laying excited states from these calculations have been found to agree with measurement, upper excited states are not expected to be correctly described. Specifically, the calculated optical properties of wurtzite $\mathrm{InN}$ [33] agree with experiment up to energies of 5.5 to $6.0 \mathrm{eV}$. The comparison of the bands from Calculations IV and V in Fig. 2 suggests that our calculated, excited states for $\mathrm{NaNO}_{2}$ could be meaningful up to $20 \mathrm{eV}$. This situation could be a feature of molecular solids.

Our calculated electron effective masses in the $\Gamma-\mathrm{X}, \Gamma-\mathrm{R}$, and $\Gamma-\mathrm{W}$ directions are $1.22,0.64$, and $0.74 \mathrm{~m}_{\mathrm{o}}$, respectively. These values clearly corroborate the anisotropic nature of this material.

Our calculations were performed at lattice constants for which experimental data are available for comparison purposes. However, the question arises as to what results could be obtained with a fully optimized crystal structure (i.e., equilibrium lattice structure). The optimization led to equilibrium lattice parameters of $3.529 \AA, 5.560 \AA$ and $5.391 \AA$ for $a, b$ and $c$, respectively. The difference between our former ( $120 \mathrm{~K}$ experimental lattice parameter) and the latter (with optimized lattice parameters) eigenvalues is approximately $0.02 \mathrm{eV}$, with the energies obtained with the experimental lattice constants being lower. The two results are practically the same as their difference is smaller than our computational uncertainty of $50 \mathrm{meV}(0.05 \mathrm{eV})$.

\section{DISCUSSION}

An added dimension of the validity of the work reported here stems from the rigor of the BZWEF method. Our calculations solved self-consistently the system of equations defining LDA. They are totally ab-initio and do not entail the use of two different theories; the sophisticated work of Zakharov et al. [35] utilized LDA wave functions as input in their quasi-particle calculations.

Unlike single trial basis set (STBS) DFT and other calculations, LDA BZW-EF results agree with experiment and point to much more physical content of LDA eigenvalues than previously thought. As explained by Zhao et al. [23], STBS calculations do not avoid a well-defined "basis set and variational effect that has plagued electronic structure calculations since their inception, with emphasis on DFT calculations.

Indeed, BZW-EF calculations begin with the minimum basis set that is just large enough to account for all the electrons in the system, i.e., $\mathrm{NaNO}_{2}$ for this work. It then augments the basis set methodically [22-24] and carries out successive, self-consistent calculations with increasingly larger basis sets. Except for the first calculation, the occupied eigenvalues of a calculation are compared numerically and graphically to those of the calculation immediately preceding it. Ultimately, the occupied eigenvalues of two consecutive calculations, say $\mathrm{N}$ and $(\mathrm{N}+1)$, are found to be identical-within computational uncertainties of about $50 \mathrm{meV}$. The results from calculation $\mathrm{N}$ provide the physical description of the system and the corresponding basis set is the optimal basis set. While Calculation $(\mathrm{N}+1)$ yields the same occupied energies as Calculation $\mathrm{N}$, some of the unoccupied energies from it are generally lower than corresponding ones from Calculation N. This extra-lowering of the unoccupied eigenvalues, for calculations with basis sets larger than the optimal one, is due to a basis set and variational effect. It is a mathematical artifact stemming from the Rayleigh theorem. For basis sets larger than the optimal one, the resulting charge density and potential are identical to those from Calculation N [23]. So, the above extra-lowering is not due to a physical interaction.

From the density of states plots, our calculated peaks of $-0.25 \pm 0.1 \mathrm{eV}$ and $-2.4 \pm 0.2 \mathrm{eV}$ in 
the valence band DOS agree with the x-ray photoelectron results of Calabrese and Heyes, [36] and the theoretical calculations of Ravindran et al. [3] and Zhuravlev and Korabel'nikov [12]. The observed sharp peak at $-0.25 \mathrm{eV}$ arises mainly from the hybridization between the $\mathrm{O} 2 p$, $\mathrm{N} 2 p$, and $\mathrm{N} 2 s$. The peak at $-2.4 \pm 0.2 \mathrm{eV}$ arises from the $\mathrm{O} 2 p$ orbitals. These observations are in agreement with that of Zhuravlev and Korabel'nikov [12] that the upper valence bands are formed by complex anion states. In the conduction bands, our calculated peaks of $3.25 \mathrm{eV}$, $8.25 \mathrm{eV}, 9.5 \mathrm{eV}, 10.50 \mathrm{eV}$ and $11.25 \mathrm{eV}$ compare favorably with the room temperature vacuum ultraviolet (VUV) data from measured values of Yamashita and Kato [37] and Ashida et al. [38], respectively.

A distinct feature of our calculated band structure in Figure 1 is the flat nature of the top of the valence band as well as that of the bottom of the conduction band. This singular feature means that the band structure of ferro- $\mathrm{NaNO}_{2}$ can well be described by localized molecular levels of an ionic crystal. This observation is similar to that of Ravindran et al. [2], Wang et al. [11] and of Jiang et al. [14]. Above the Fermi energy, as can be seen from the DOS, many conduction bands exist and some are highly dispersed. From the pDOS for the upper valence and the conduction bands, we can infer that the Na states are high up in the conduction bands and do not partake in the formation of the molecular solid.

The top-most valence bands and bottom-most conduction bands are flat. Consequently, the exact maximum and minimum values of the valence and conduction bands, respectively, cannot be easily identified by merely looking at the plots. From our outputs, before setting the top of the valence bands equal to zero, we found that the maximum of the valence band (VBM) is at the $W$ point while the minimum of the conduction band (CBM) is at $R$, respectively, giving a fundamental, indirect energy band gap of $2.83 \mathrm{eV}$ from Calculation IV. Our calculated direct gaps are $2.90,2.98,3.02,3.22$, and $3.51 \mathrm{eV}$ at $R, W, X, \Gamma$, and $T$, respectively. The scanning electron microscopy measurements of Balabinskaya et al. [9] reported a direct band gap of 3.0 $\mathrm{eV}$.

The extent to which our electronic structure calculations take temperature into account is practically limited to the use of lattice constants obtained at a given temperature. With the lattice constants obtained at $120 \mathrm{~K}$, our calculated direct gap of $3.22 \mathrm{eV}$ compares favorably with the infrared absorption result of $3.22 \mathrm{eV}$ obtained by Sidman [6] at a low temperature (77 $\mathrm{K})$. Predictably, it is larger than the room temperature $(293.15 \mathrm{~K})$ measurement of $3.14 \mathrm{eV}$ of Verkhovskaya and Sonin [7].

The positions of our VBM (at $W$ ) and CBM (at $R$ ) are the same as those from the work of Ravindran et al. [11] that found a calculated, indirect band gap of $2.2 \mathrm{eV}$. Jiang et al. [14] calculated an indirect gap of $2.95 \mathrm{eV}$. However, these authors obtained their VBM at $S$. In our calculations, only the $1 s$ states of $N a, N$, and $O$, respectively, were in the core. Jiang et al. [14] placed $1 s$, $2 s$, and $2 p$ electrons of sodium in the core.

Our calculated electron effective masses of $1.18,0.63$, and $0.73 \mathrm{~m}_{\mathrm{o}}$ in the $\Gamma-\mathrm{X}, \Gamma-\mathrm{R}$, and $\Gamma-$ $\mathrm{W}$ directions, respectively, show the highly anisotropic nature of this material. We know of no experimental or calculated electron effective masses for $\mathrm{NaNO}_{2}$, except the semi-empirical, temperature dependent result of El-Dib and Hassan [8] who found an electron effective mass of $0.61 \mathrm{~m}_{\mathrm{o}}$ in the $\Gamma-\mathrm{R}$ direction. Our result of 0.63 , from $\Gamma-\mathrm{R}$, agrees with their finding. 


\section{CONCLUSION}

The above results and related discussions are expected to add to our understanding of ferro$\mathrm{NaNO}_{2}$. The noted agreement between our calculated results and experiment will hopefully motivate further experimental and theoretical studies of this material. Our calculated eigenvalues at high symmetry points should aid in some comparison with future studies.

This work and similar ones from this group [17-24] indicate that LDA can correctly describe and predict electronic and related properties of semiconductors, provided one methodically search for the optimal basis set that minimizes the occupied energies. The predictive capability of such calculations is expected to inform and to guide the design and fabrication of semiconductor based devices. For example, for binary to quaternary systems, theoretical studies of variations of the concentration of an element (or more) have the potential to impact positively industrial activities.

\section{Acknowledgments}

This work was funded in part by the National Science Foundation and the Louisiana Board of Regents (Award Nos. EPS-1003897, and NSF (2010-15)-RII-SUBR, 0754821, and HRD-1002541), the Louisiana Optical Network Initiative (LONI, SUBR Award No. 2-10915). CEE wishes to thank Govt. of Ebonyi State, Nigeria.

\section{References}

1. S. Sawada, S. Nomura, S. Fuji, and I. Yoshida, Phys. Rev. Letts. 1, 320 (1958).

2. P. Ravindran, A. Delin, B. Johansson, and O. Eriksson, Phys. Rev B 59, 1776 (1999).

3. M. Kamada, M. Yoshikawa, and R. Kato, J. Phys. Soc. Jpn. 39, 1004 (1975).

4. Y. Asao, I. Yoshida, R. Ando, and S. Sawada, J. Phys. Soc. Jpn., 17, 442 (1962).

5. Y. Takagi and K. Gesi, J. Phys. Soc. Jpn, 22, 979 (1967).

6. J.W. Sidman, J. Am. Chem. Soc. 79, 2669 (1957).

7. K.A. Verkhovskaya and A.S. Sonin, Soc. Phys-JETP 25, 249 (1967).

8. A.M. El-Dib and H.F. Hassan, Phys. Stat. Sol. B 126, 587 (1984).

9. A. S. Balabinskaya, E. N. Ivanova, M. S. Ivanova, Yu. A. Kumzerov, S. V. Pan'kova, V. V. Poborchii, S. G. Romanov, V. G. Solovyev, and S. D. Khanin, Glass Phys. and Chem. 31 (3), 330-336 (2005).

10. K. -S Kam and J.H. Henkel, Phys. Rev. B 17, 1361 (1978). 
11. Y.X. Wang, W.L. Zhong, C.L. Wang, P.L. Zhang, and Y.P. Peng, Phys. Letts. A 269, 252-256 (2000); ibid, Solid State Comm. 112 495-498 (1999).

12. Y. N. Zhuravlev and D. V. Korabel'nikov, Phys. of the Solid State, 51 (1), 69-77 (2009).

13. Y.N. Zhuravlev and A. S. Poplavnoi, Russian Phys. J., 44 (12) (2001).

14. H. Jiang, Y.N. Xu, and W. Y. Ching, Ferroelectrics, 136 (1), 137-146 (1992).

15. Maureen I. McCarthy, K. A. Peterson, and W.P. Hess, J. Phys. Chem. 100, 6708-6714 (1996).

16. J. H. Henkel, T. C. Collins, J. L. Iveyt, and R. N. Euwema, Int. J. of Quant. Chem. 9, $529-533(2009)$.

17. D Bagayoko, G L Zhao, J D Fan, and J T Wang, J. Phys.: Condens. Matter 105645 (1998).

18. E. C. Ekuma and D. Bagayoko, Jpn. J. Appl. Phys. 50, 101103 (2011).

19. E. C. Ekuma, L. Franklin, J. T. Wang, G. L. Zhao, and D. Bagayoko, Can. J. Phys. $8 \boldsymbol{9}$ (3), pg. 319-324 (2011). See also: E. C. Ekuma, L. Franklin, J. T. Wang, G. L. Zhao, and D. Bagayoko, Physica B, 406 (8), pg. 1477-1480 (2011).

20. D. Bagayoko and G. L. Zhao, Physica C 364-365, Pages 261-264 (2001).

21. D. Bagayoko, L. Franklin, and G. L. Zhao, J. Appl. Phys. 96, 4297-4301 (2004); D. Bagayoko, L. Franklin, G. L. Zhao, and H. Jin, J. Appl. Phys. 103, 096101 (2008).

22. C. E. Ekuma, M. Jarrell, J. Moreno, and D. Bagayoko, AIP Advances 2, 012189 (2012).

23. G. L. Zhao, D. Bagayoko, and T. D. Williams. Physical Review B60, 1563, 1999.

24. D. Bagayoko. Proceedings, International Seminar on Theoretical Physics and Applications to Development (ISOTPAND), August 2008, Abuja, Nigeria. Available in the African Journal of Physics (http://sirius-c.ncat.edu/asn/ajp/allissue/ajp-ISOTPAND/index. $\mathrm{html}$ ).

25. D. M. Ceperley and B. J. Alder, Phys. Rev. Lett. 45, 566 (1980).

26. S. H. Vosko, L. Wilk, and M. Nusair, Can. J. Phys. 58, 1200 (1980).

27. B. N. Harmon, W. Weber, and D. R. Hamann, Phys. Rev. B 25, 1109 (1982).

28. W. Kohn and L. J. Sham, Phys. Rev. 140, A1133 (1965).

29. G.E. Zeigler, Phys. Rev. 38, 1040 (1931).

30. International Tables for Crystallography, Vol. A: Space-Group Symmetry, $5^{\text {th }}$ Edn. Theo Hahn, Ed. Springer (2005). 
31. M. Okuda, S. Ohba, Y. Saito, T. Ito, and I shibya, Acta Cryst. B 46, 343 (1990) in, as quoted in Inorganic Crystal Structure Database (ICSD), NIST, Release 2010/1.

32. M. Kamada, K. Ichikawa and K. Tsutsumi, J. Phys. Soc. Jpn. 50 (1), 170 (1981).

33. H. Jin, G. L. Zhao, and D. Bagayoko, J. Appl. Phys. 101, 033123 (2007).

34. D. Bagayoko, L. Franklin, and G. L. Zhao, Phys. Rev. B 76, 037101 (2007)

35. O. Zakharov, A. Rubio, X. Blase, M. Cohen, and S. Louie, Phys. Rev. B 50, 10780 (1994).

36. A. Calabrese and R. G. Hayes, J. Electron Spectrosc. Relat. Phenom. 6(1), 1 (1975).

37. A. Yamashita and R. Kato, J. Phys. Soc. Jpn. 29 (6), 1557 (1970).

38. M. Ashida, O. Ohta, M. Kamada, M. Watanabe, and R. Kato, J. Electron Spectrosc. Relat. Phenom. 79, 55 (1996). 